\title{
Silencing of histone methyltransferase NSD3 reduces cell viability in osteosarcoma with induction of apoptosis
}

\author{
ZHIWEI LIU $^{1^{*}}$, LIANHUA PIAO ${ }^{2 *}$, MING ZHUANG $^{1}$, XUBIN QIU $^{1}$, XIAOSHUANG XU ${ }^{2}$, \\ DAWEI ZHANG ${ }^{2}$, MENGMENG LIU $^{2}$ and DING REN ${ }^{3,4}$
}

\author{
${ }^{1}$ Department of Orthopaedics, The Third Affiliated Hospital of Soochow University, Changzhou, Jiangsu 213000; \\ ${ }^{2}$ Institute of Bioinformatics and Medical Engineering, Jiangsu University of Technology, Changzhou, Jiangsu 213001; \\ ${ }^{3}$ Out-Patient Department, PLA No. 85th Hospital, Shanghai 200006; ${ }^{4}$ Department of Orthopaedics, \\ PLA No. 100th Hospital, Suzhou, Jiangsu 215007, P.R. China
}

Received March 10, 2017; Accepted August 10, 2017

DOI: $10.3892 /$ or.2017.5936

\begin{abstract}
NSD3 is a histone lysine methyltransferase that methylates histone $\mathrm{H} 3$ at lysine 36 . NSD3 is located at chromosome $8 \mathrm{p} 11.23$, the locus that exhibits strong cancer relevance. Thus, NSD3 is likely involved in multiple human cancers. Nevertheless, its roles in human carcinogenesis remain unknown. In the present study, we demonstrated that silencing of NSD3 in osteosarcoma, the most common primary bone cancer in children and adolescents, results in a marked decrease in the number of viable cancer cells, accompanied by increases in the cell population at the G2/M phase and the number of apoptotic cells. In addition, 549 NSD3-regulated genes were identified and a set of selected candidate genes were validated. Bioinformatic analysis revealed that NSD3 negatively regulates a number of genes that are involved in the process of negative regulation of signal transduction as well as negative regulation of signaling and cell communication. Our results indicate the oncogenic roles of NSD3 in the development and progression of human osteosarcoma, and implicate NSD3 as a potential molecular target for selective therapy for human osteosarcoma.
\end{abstract}

\section{Introduction}

Osteosarcoma (OS), a mesenchymal tumor histologically characterized by the presence of malignant mesenchymal cells which produce osteoid, is the most common primary malignancy of bone, particularly with a high incidence rate in children and young adults (1). After conventional treatment, consisting of the combination of pre- and post-operative

Correspondence to: Dr Lianhua Piao, Institute of Bioinformatics and Medical Engineering, Jiangsu University of Technology, 1801 Zhongwu Avenue, Changzhou, Jiangsu 213001, P.R. China

E-mail: piaolianhua@jsut.edu.cn

*Contributed equally

Key words: NSD3, osteosarcoma, histone methyltransferase, human carcinogenesis, epigenetics chemotherapy with surgical removal of the tumor, the 5-year overall survival rate approaches $60-70 \%$ (2-4). Nonetheless, $\sim 30-35 \%$ patients, with successful resection and adjuvant chemotherapy, still develop metastases $(2,5)$. There has been no substantial improvement in the long-term outcomes for patients presenting with localized or disseminated disease over the last three decades. Thus, identification and validation of novel targetable agents as adjuvant to conventional chemotherapeutics to provide better control of osteosarcoma is absolutely important and urgent.

Osteosarcoma is a heterogeneous tumor with a diversity of genetic changes. To date, the heterogeneity of osteosarcoma has been reported through whole genome sequencing approaches $(6,7)$, while unfortunately, there has been no significant improvement in molecularly targeted therapies that may benefit osteosarcoma patients. Hence, identifying potential targetable genetic aberrations or pathway alterations may lead to the development of targeted therapies for osteosarcoma.

NSD3 (also known as WHSC1L1), a Su(var)3-9, enhancerof-zeste and trithorax (SET) domain containing histone lysine methyltransferase, can dimethylate and trimethylate histone $\mathrm{H} 3$ at lysine 36 (8-11). NSD3, the third member of the NSD family, shares a C-terminal block of $\sim 700$ amino acids with the other two family members, NSD1 (also known as SOTOS) and NSD2 (also referred to as WHSC1 and MMSET) which also methylate H3K36. In addition to the catalytic SET domain with pre- and post-SET domains, NSD3 possesses two PWWP (the conserved sequence motif of Pro-Trp-Trp-Pro) domains and five PHD (plant homeodomain) fingers, both of which are often involved in chromatin-associated biological processes through crosstalks with histone and DNA reader or modifier proteins. Hence, NSD3 is involved in a variety of biological processes such as chromatin modification, transcriptional regulation, and DNA repair either through direct regulation of histone methylation or through the protein-protein interactions by these specific domains. There are two major isoforms of NSD3, the long full-length isoform and the short isoform lacking the SET domain and only possessing the first $\mathrm{N}$-terminal PWWP domain.

NSD3, as a nuclear protein, is located at chromosome $8 \mathrm{p} 11.23$, the locus that exhibits strong cancer relevance. 
Indeed, NSD3 is likely involved in solid and hematological tumors (12-17), and functions as an oncogene in the development and progression of cancer (18-20). In midline carcinoma, a novel NSD3-NUT fusion oncogene containing N-terminal region of NSD3 has been demonstrated (21). Notably, the NSD3 portion of the fusion protein lacks the catalytic SET domain and contains only its first PWWP domain. In addition, NSD-short, possessing only the N-terminal PWWP domain, also couples BRD4 to the CHD8 chromatin remodeler and sustains acute myeloid leukemia (AML) cell proliferation (22). These findings indicate the importance of these specific nuclear domains in NSD3. NSD3 was reported as a translocation partner of NUP98 in an AML patient associated with $\mathrm{t}(8 ; 11)(\mathrm{p} 11.2 ; \mathrm{p} 15)(23,24)$. NSD3 is also essential for neural crest gene expression during specification (25). Collectively, all of these studies suggest the important role of NSD3 in human carcinogenesis. However, the molecular mechanisms of NSD3 in human carcinogenesis are still obscure and remain to be clarified.

In the present study, we demonstrated that inhibition of NSD3 in osteosarcoma cells caused a marked reduction in cancer cell viability and survival, with an increase in the proportion of cells at the G2/M phase and the number of apoptotic cells. Additionally, a set of NSD3-regulated genes involved in multiple biological processes were identified by RNA-seq analysis. Thus, these results demonstrated the oncogenic functions of NSD3 in osteosarcoma, and furthermore, indicate that NSD3 could be a promising target for the treatment of osteosarcoma.

\section{Materials and methods}

Cell lines and cell culture. Human osteosarcoma cell lines HOS, U2OS and MG-63 were purchased from the Shanghai Institute for Biological Sciences, Chinese Academy of Cell Resource Center (Shanghai, China). HOS, U2OS and MG-63 cells were grown in minimum essential medium (MEM; Gibco, Shanghai, China), supplemented with $10 \%$ fetal bovine serum (Beijing Yuanheng Shengma Research Institution of Biotechnology, Beijing, China) and 1\% antibiotic/antimycotic solution (Gibco) at $37^{\circ} \mathrm{C}$ in a humidified incubator with $5 \% \mathrm{CO}_{2}$.

RNA extraction and real-time PCR. Total RNA was isolated from the cells using an EASYspin Plus Tissue/Cell RNA extraction kit (Aidlab Biotechnologies Co., Ltd., Beijing, China). RNA was reverse transcribed to cDNA using the ThermoScript First Strand cDNA Synthesis kit (Aidlab Biotechnologies Co., Ltd.) according to the manufacturer's instructions. Semi-quantitative reverse transcription-PCR was performed using KOD-Plus-Neo DNA polymerase (Toyobo Biotech Co., Ltd., Shanghai, China) to investigate the complete knockdown of NSD3. Each PCR regime consisted of initial denaturation at $94^{\circ} \mathrm{C}$ for $2 \mathrm{~min}$ followed by 22 cycles (for ACTB), 32 cycles (for NSD3) at $98^{\circ} \mathrm{C}$ for $10 \mathrm{sec}, 55^{\circ} \mathrm{C}$ for $30 \mathrm{sec}$ and $68^{\circ} \mathrm{C}$ for $45 \mathrm{sec}$. The primer sequences were: 5'-TTGGCTTGACTCAGGATTTA-3' and reverse 5'-ATGCTA TCACCTCCCCTGTG-3' for $\beta$-actin $(A C T B)$; and 5'-CCA TGCAGAGAAAGCATTGA-3' and 5'-TCTTCCTCTTCC GCACTTGT-3' for NSD3. qRT-PCR was conducted using SYBR Premix Ex $\operatorname{Taq}^{\mathrm{TM}}$ (Takara, Dalian, China) at $95^{\circ} \mathrm{C}$ for
$30 \mathrm{sec}$, followed by 40 cycles of $95^{\circ} \mathrm{C}$ for $5 \mathrm{sec}$ and $60^{\circ} \mathrm{C}$ for $34 \mathrm{sec}$ in the ABI StepOnePlus Real-time PCR system. Relative gene expression was quantified relative to the GAPDH level using the comparative cycle threshold $(\mathrm{Ct})$ method. Each sample was analyzed in duplicate.

Cell viability and colonyformation assays. Cells $\left(10^{4}\right.$ cells/well $)$ were plated in 96-well plates the day before treatment to allow cell attachment, and transfected with NSD3-specific siRNA duplex (siNSD3\#1, 5'-CUCACAAAUGGGUAUCCAU-3'; siNSD3\#2, 5'-GUACUGAAAUUCGGAGACA-3'); or EGFP siRNA duplex (5'-GCAGCACGACUUCUUCAAG-3') as a negative control, respectively, using Lipofectamin RNAiMAX (Invitrogen Life Technologies, Shanghai, China) according to the manufacturer's recommendations. Cell viability was measured using Cell-Counting Kit-8 (Dojindo, Shanghai, China) $96 \mathrm{~h}$ after transfection. The absorbance was measured at a wavelength of $450 \mathrm{~nm}$, and the percentage of cell viability was calculated as the percentage of absorption. Each sample was assayed in triplicate. All experiments were performed in triplicate and repeated two times independently. For the colony formation assay, the cells were seeded into 6-well plates $\left(2 \times 10^{3}\right.$ cells/well) and treated with the specific siRNA for 4-8 h, respectively, followed by a medium refresh. After 10 days for HOS, U2OS and 14 days for MG-63, the cells in the plates were stained with crystal violet solution.

Annexin V-FITC and propidium iodide (PI) staining. Cells treated with the specific siRNA for $96 \mathrm{~h}$ were centrifuged and collected. The cell pellets were washed with phosphate-buffered saline (PBS) and resuspended in 1X Annexin-binding buffer with $5 \mu \mathrm{l}$ of FITC Annexin V and $5 \mu \mathrm{l}$ PI (both from BD Pharmingen, San Diego, CA, USA). After a 15-min incubation at room temperature in the dark, the samples were analyzed by BD FACSAria II. FITC ${ }^{+} / \mathrm{PI}^{-}$and $\mathrm{FITC}^{+} / \mathrm{PI}^{+}$cells were considered as early and late apoptotic cells and analyzed. The percentage of apoptotic cells in total cells was designated as the apoptotic index. For cell cycle analysis, $96 \mathrm{~h}$ after the transfection, the cells were fixed with $70 \%$ ethanol at $4^{\circ} \mathrm{C}$, incubated with $500 \mu \mathrm{l}$ of PBS containing $0.5 \mathrm{mg}$ of RNase at $37^{\circ} \mathrm{C}$ for $30 \mathrm{~min}$, and finally, cells stained with $50 \mu \mathrm{g} / \mathrm{ml} \mathrm{PI}$ were analyzed by fluorescence-activated cell sorting (FACS).

RNA-seq analysis. Indexed libraries from HOS cells incubated with siEGFP or siNSD3 were subjected to RNA-seq on the Illumina HiSeq 2000 platform. Gene expression was quantified using the fragments per kilobase of transcript per million mapped reads (FPKM) normalization method (26), and Cufflinks was used for FPKM quantification. Gene expression and differential transcription between siEGFP- and siNSD3-treated cells were evaluated using Cuffdiff. The lists of significantly differentially expressed genes were obtained with the threshold of $p$-value $\leq 0.05$ and a fold-change $\geq 2$. Gene ontology (GO) analysis was performed in the standard enrichment computation method.

\section{Results}

Silencing of NSD3 attenuates osteosarcoma cell viability. To investigate the biological significance of NSD3 in 

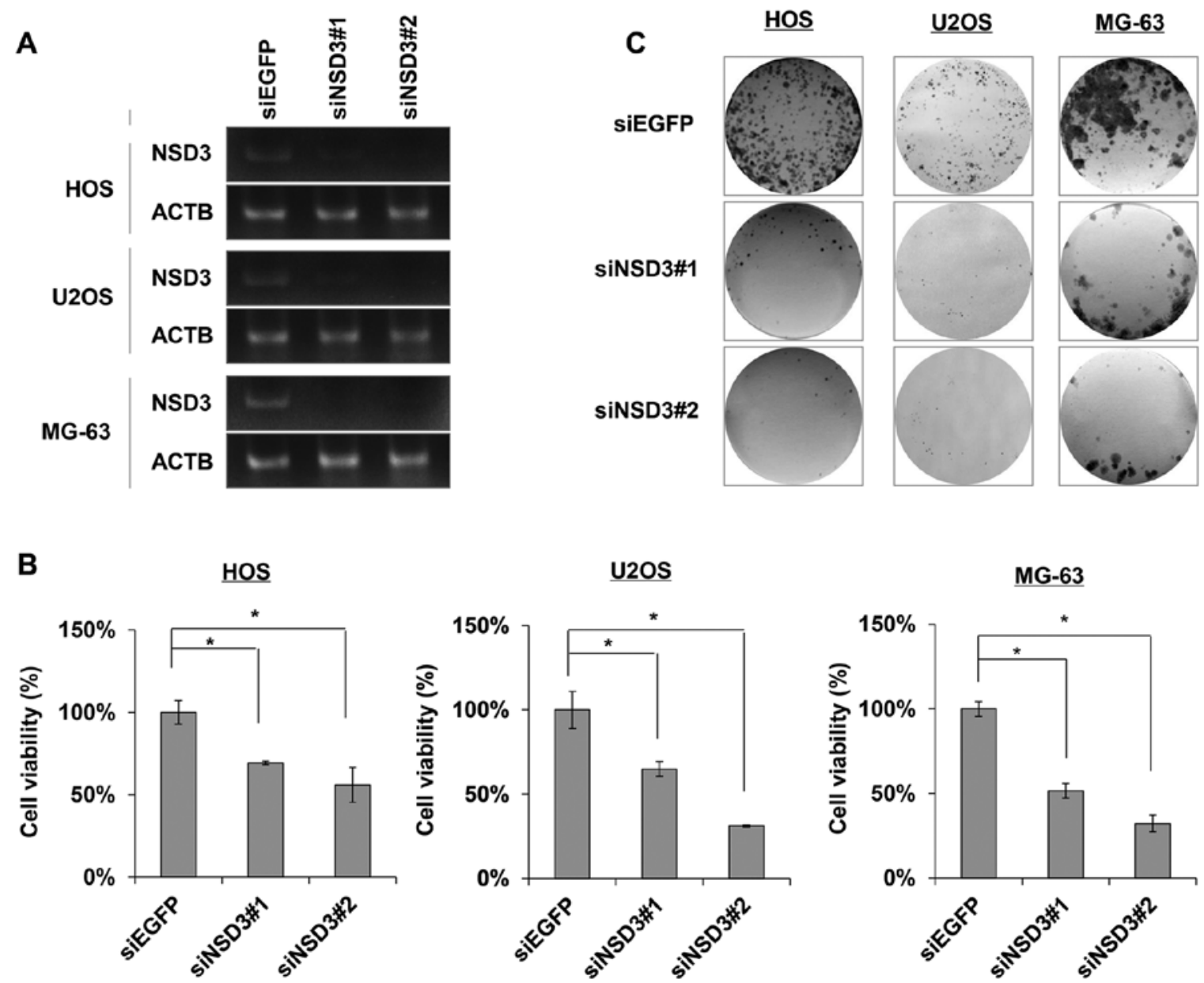

Figure 1. Effect of NSD3 on the growth of osteosarcoma cells. (A) Semi-quantitative RT-PCR was used to examine the knockdown effect of NSD3 expression on osteosarcoma cells (HOS, U2OS and MG-63 cells) transfected with an siRNA specific to NSD3 (siNSD3\#1 and siNSD3\#2) or control siRNA (siEGFP). (B) NSD3-depleted osteosarcoma cancer cells showed a drastic reduction in cell viability. Results of the MTT assay were assessed at $96 \mathrm{~h}$ after transfection. The experiments were carried out in triplicate, and each average is plotted with error bars indicating the standard deviation (SD) ( ${ }^{*} \mathrm{p}<0.05$, Student's t-test). (C) Colony formation assays with osteosarcoma cells after NSD3 knockdown. Cells were stained with $0.1 \%$ crystal violet 10 days (for HOS and U2OS) or 14 days (for MG-63) after treatment with the specific siRNA.

osteosarcoma cells, the HOS, U2OS and MG-63 cancer cell lines were transfected with an NSD3-specific siRNA duplex (siNSD3\#1, siNSD3\#2) or EGFP siRNA duplex (siEGFP) as a negative control, respectively. Results of semi-quantitative RT-PCR confirmed the complete knockdown effects of NSD3 expression in the cells transfected with siNSD3\#1 or siNSD3\#2 for $96 \mathrm{~h}$ compared with the siEGFP control (Fig. 1A). Cell viability assay data revealed a marked reduction in the number of viable cells in the siNSD\#1 or siNSD3\#2-treated cancer cells as compared to the siEGFP control, reaching a $30-50 \%$ reduction in the HOS, U2OS and MG-63 osteosarcoma cells (Fig. 1B). The reduction in viable cells upon NSD3 knockdown was investigated with two different siRNA sequences specific for NSD3, indicating it was not due to off-target effects of the siRNA. Colony formation assay was conducted to assess the effect of NSD3 on the proliferation of cancer cells, and as a result, siNSD3\#1- or siNSD3\#2-treated cancer cells formed much fewer and smaller colonies than the siEGFP-treated cancer cells (Fig. 1C), probably due to cell death caused by apoptosis and cell cycle-related events. These findings elucidated the oncogenic functions of NSD3 in the development and progression of osteosarcoma.
Knockdown of NSD3 induces apoptosis of cancer cells. Given the drastic reduction in viable cells following NSD3 silencing, we evaluated the effects of NSD3 on cell apoptosis using Annexin V-FITC and PI staining followed by FACS analysis. In the scatter plot of double variable events, cells in quadrant Q4 (FITC $\left./ \mathrm{PI}^{-}\right)$were considered viable, cells in quadrant Q2 $\left(\mathrm{FITC}^{+} / \mathrm{PI}^{+}\right)$were in the late apoptotic stage, and cells in quadrant Q3 (FITC $/ \mathrm{PI}^{-}$) represented early apoptotic cells. As a result, a striking increase was observed in both the early and late stage of apoptosis in the NSD3-depleted HOS, U2OS and MG-63 osteosarcoma cells compared with the control cells (Fig. 2A). The number of apoptotic cancer cells was increased 3.3-fold in the NSD3-depleted HOS cells, 5.8-fold in the U2OS cells, and 4.7-fold in the MG-63 osteosarcoma cells (Fig. 2B), showing that silencing of NSD3 induced apoptosis in all three measured osteosarcoma cell lines. These data suggest that NSD3 may contribute to cancer cell apoptosis.

Depletion of NSD3 increases the proportion of cells in the G2/M phase. Since deletion of NSD3 decreased cell viability and induced cell apoptosis, we hypothesized that NSD3 is also involved in the cell cycle. To assess the roles of NSD3 


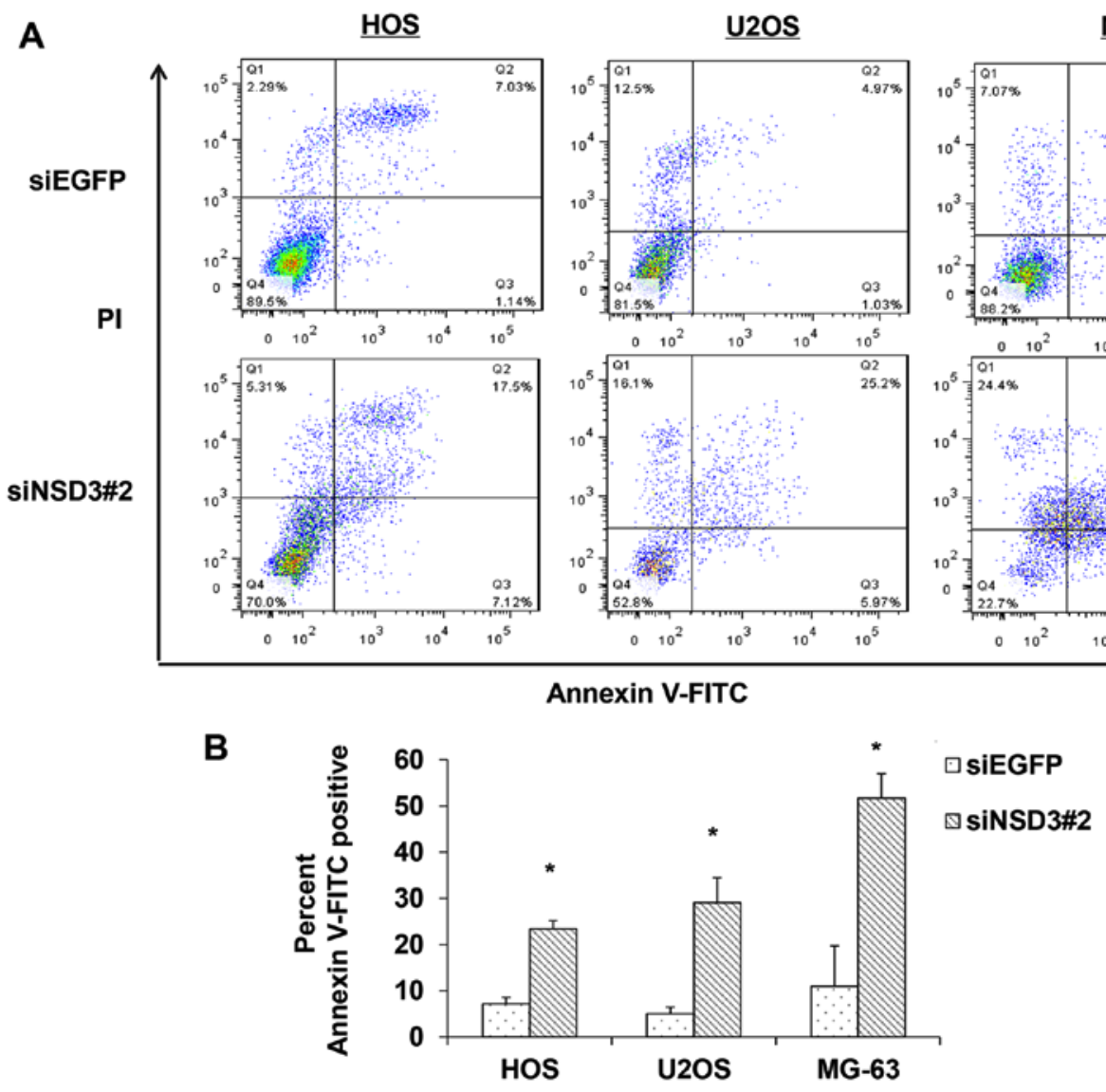

Figure 2. Assessment of apoptosis in osteosarcoma cells. (A) The apoptotic rates were detected using flow cytomertry with Annexin V-FITC/PI dual staining. NSD3-depleted (siNSD3\#2) or control (siEGFP) cells were double stained with dual Annexin V-FITC and propidium iodide (PI). Flow cytometry profile representing Annexin V-FITC on the x-axis and PI on the y-axis. Quadrant Q1 (upper left, FITC-/PI') displays necrotic cells, quadrant Q2 (upper right, FITC ${ }^{+} /$ $\mathrm{PI}^{+}$) displays late apoptotic cells, quadrant Q3 (lower right, $\mathrm{FITC}^{+} / \mathrm{PI}^{-}$) shows early apoptotic cells and quadrant $\mathrm{Q} 4$ (lower left, $\mathrm{FITC}^{-} / \mathrm{PI}$ ) shows live cells (B) The percentage of total apoptotic cells $(\mathrm{Q} 2+\mathrm{Q} 3)$ was calculated and is shown in the histogram. The experiment was repeated two times and data represent the average percentage of apoptotic cells (" $\mathrm{p}<0.05$, Student's t-test).

in the progression of the cell cycle, viable cells treated with siRNA specific to NSD3 or control were collected, fixed with ethanol and stained with PI for flow cytometric analysis. A 2-fold increase in the population of G2/M phase was found in the NSD3-depleted HOS cells (Fig. 3A). Concordant increases were obtained in the other two osteosarcoma cancer cell lines U2OS and MG-63. Furthermore, apart from the marked increases in the $\mathrm{G} 2 / \mathrm{M}$ population, slight increases in the $\mathrm{S}$ phase population were also observed in the NSD3-depleted U2OS and MG-63 cells (Fig. 3A). Data from three independent experiments were analyzed and the average population of each phase was calculated (Fig. 3B). Consistent alterations in G2/M populations upon NSD3 silencing were observed. These findings imply that knockdown of NSD3 leads to G2/M cell cycle arrest and NSD3 is likely to be involved in the regulation of the G2/M checkpoint.

Identification of downstream genes of NSD3 by RNA-seq analysis. To identify how NSD3 contributes to the development and progression of osteosarcoma, we searched NSD3-regulated genes in HOS cells on a genome-wide scale. RNA sequencing gene expression analysis was performed in HOS cells treated with NSD3-specific siRNA (siNSD3\#2) or EGFP-specific siRNA (siEGFP) as a control. A variety of genes were identified, including 244 upregulated genes and 305 downregulated genes in the NSD3-depleted HOS cells with the threshold of $\mathrm{p} \leq 0.05$ and a fold-change $\geq 2$ as compared with the control (Fig. 4A), indicating that NSD3 is correlated with both gene activation and repression. GO term enrichment analysis of the biological process category revealed that genes with increased expression after NSD3 depletion were involved in various biological processes, such as negative regulation of signal transduction, negative regulation of signaling, negative regulation of cell communication, phosphorus metabolic process, negative regulation of response to stimulus (Fig. 4B), whereas genes with decreased expression after NSD3 depletion were involved in negative regulation of interleukin-6 production and intracellular sequestering of iron ion (Fig. 4C). These findings, together with the effect of NSD3 on cancer cell proliferation and cell apoptosis, provide evidence that NSD3 is an oncogenic driver in osteosarcoma. To validate the findings of RNA-seq, the top 20 upregulated and downregulated genes were selected to be analyzed by quantitative real-time PCR. Moreover, to exclude the set of off-target genes, the results were confirmed with two different NSD3-specific siRNAs, siNSD3\#1 and siNSD3\#2, respectively (Fig. 5A). As a result, 14 upregulated and 12 downregulated genes by depletion of NSD3 were identified. Herein, the top 10 upregulated (Fig. 5A) and downregulated (Fig. 5B) genes are listed, including tumor-related genes, such as TRPM8, LAPTM5, ITGA2, BMF, CAPG and CMTM3. 
A

HOS

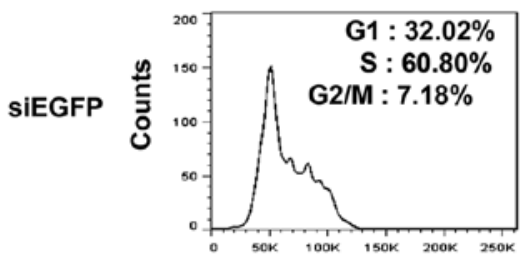

PI

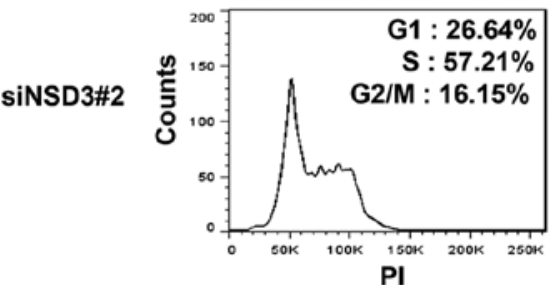

HOS

\section{B}

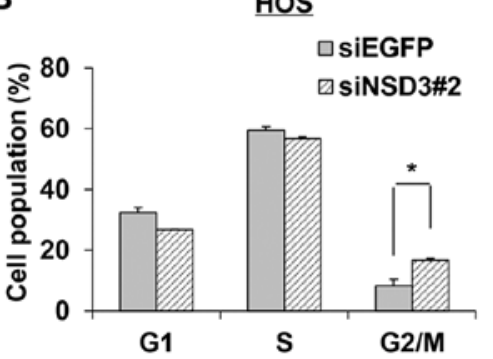

U2OS

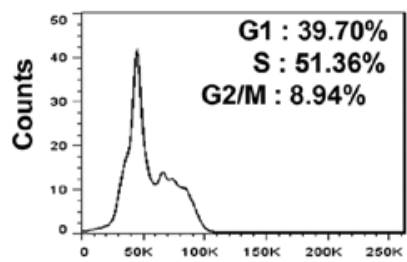

Pl

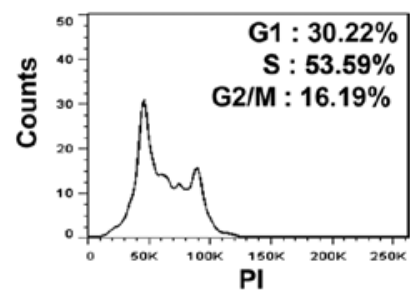

U2OS

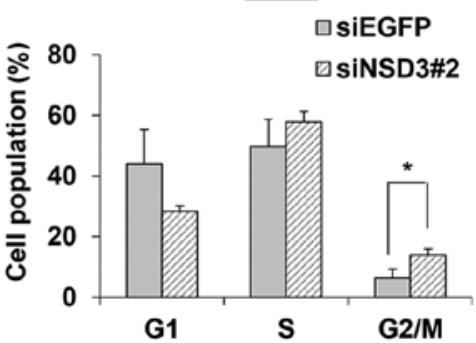

MG-63

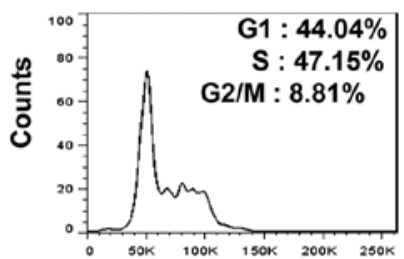

PI

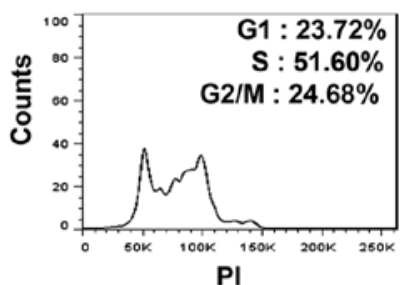

MG-63

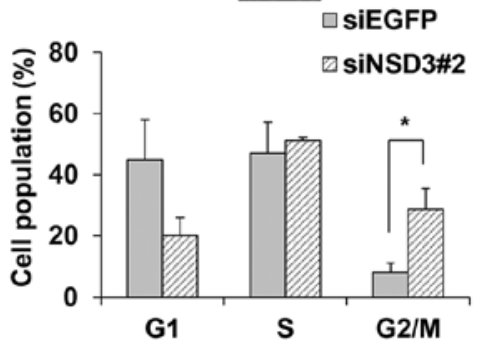

Figure 3. Depletion of NSD3 leads to an increase in the G2/M phase population. (A) Propidium iodide (PI) staining of DNA detected by FACS of osteosarcoma cells treated with siRNA specific for NSD3 (siNSD3\#2) or control EGFP (siEGFP) for 96 h. The percentage of cells in each group was calculated using ModFit LT software. (B) The average percentages of the cell population in each phase (G1, S and G2/M) of the cell cycle from three independent experiments were calculated. Mean \pm SD of three independent experiments. p-values were calculated using Student's t-test; ${ }^{*}$ p $<0.05$

A

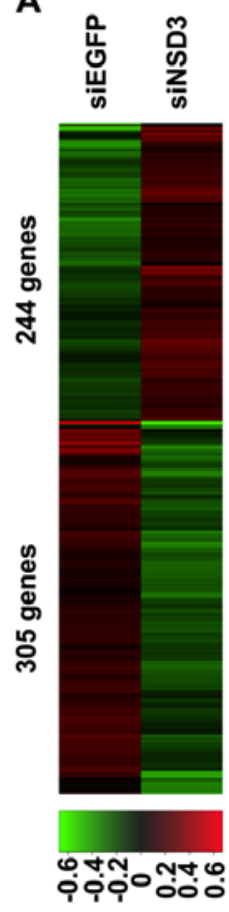

B Genes with upregulated after NSD3 knockdown:

Negative regulation of signal transduction Negative regulation of signaling Negative regulation of cell communication Phosphorus metabolic process Phosphate-containing compound metabolic process Negative regulation of response to stimulus Regulation of cellular component movement Anatomical structure morphogenesis Protein phosphorylation Regulation of locomotion

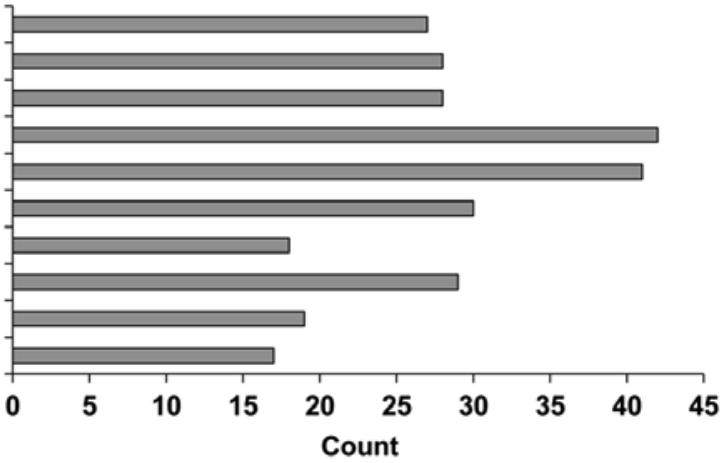

C Genes with downregulated after NSD3 knockdown:

Negative regulation of interleukin-6 production Intracellular sequestering of iron ion Sequestering of iron ion Regulation of high voltage-gated calcium channel activity Response to follicle-stimulating hormone

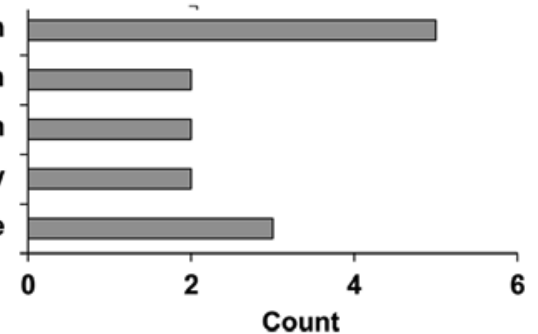

Figure 4. Downstream genes of NSD3 were identified through RNA-seq. (A) Heat map of upregulated and downregulated genes following depletion of NSD3. RNA sequence analyses were conducted with HOS cells treated with NSD3-specific siRNA or EGFP control siRNA for $96 \mathrm{~h}$. A heat map of genes regulated by NSD3 in HOS cells is shown. (B) Analysis of Gene ontology (GO) term enrichment of the biological process category of genes with increased expression after NSD3 depletion. Top $10 \mathrm{GO}$ terms ranked according to the p-value are plotted. (C) Gene ontology analysis of the biological process category of genes with decreased expression after NSD3 depletion. Top five GO terms ranked according to the p-value are plotted. 

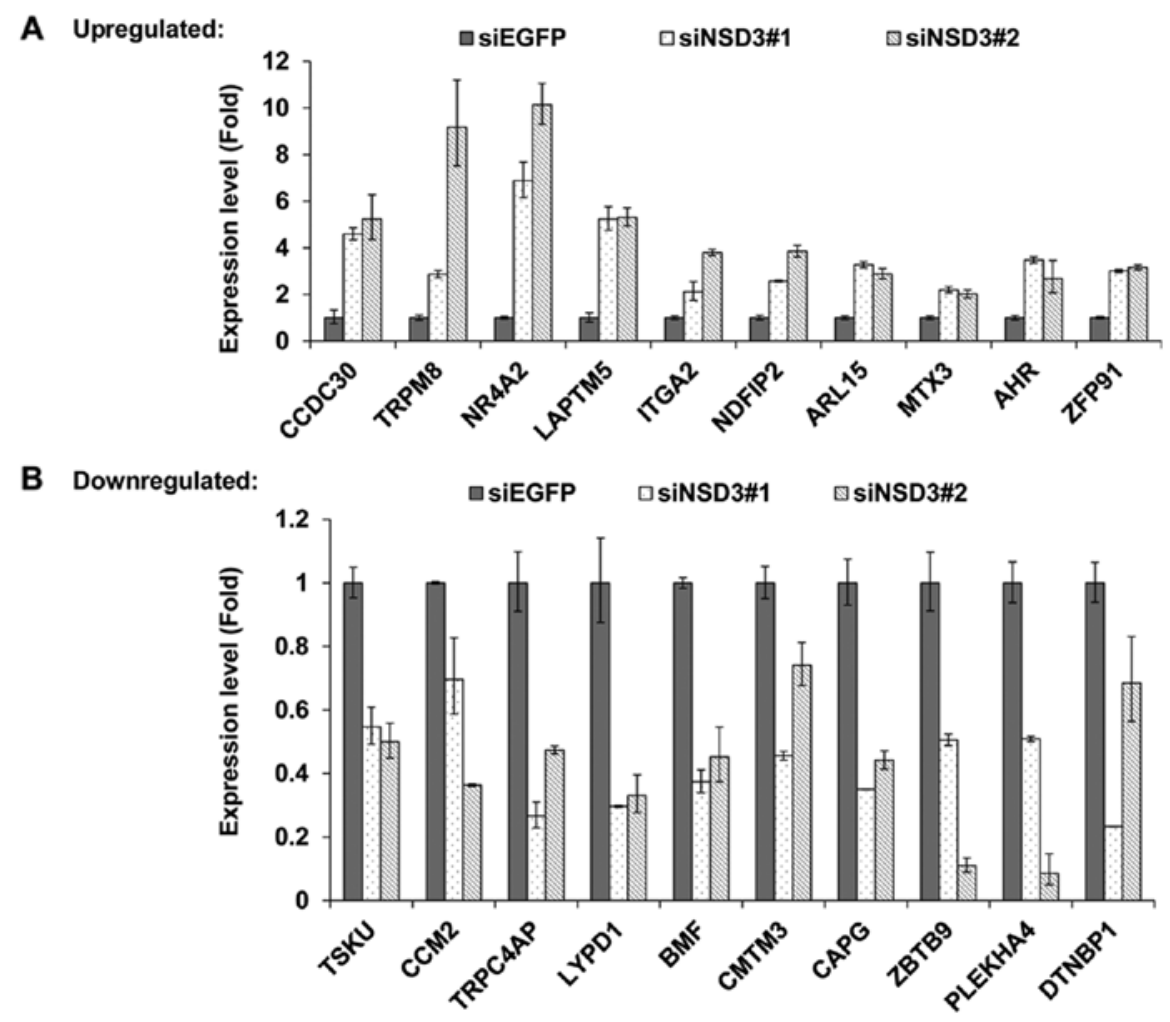

Figure 5. Validation of RNA-seq results via quantitative real-time PCR. Top 20 upregulated and downregulated genes after NSD3 silencing ranked according to the fold-change were selected for further confirmation. Expression of selected NSD3-associated genes [upregulated (A) or downregulated (B)] upon NSD3 knockdown was measured in control (siEGFP) and NSD3-depleted (siNSD3\#1 and siNSD3\#2) HOS cells by quantitative real-time PCR analysis. The relative mRNA quantity (normalized to GAPDH) in siNSD3\#1 and siNSD3\#2 samples was compared to that in the control siEGFP sample. Primers designed for each of the genes were used to perform qPCR analysis. Error bars represent SEM.

\section{Discussion}

Epigenetic modifications, including DNA methylation, covalent histone modifications and nucleosome positioning, play critical roles in normal mammalian development and regulation of gene expression, and are believed to be ubiquitous drivers of human carcinogenesis. Histone methyltransferases, the key group in the regulation of histone methylation, are commonly disrupted in cancer and contribute to human carcinogenesis. Hence, histone methyltransferases have emerged as crucial targets for the treatment of cancer in both industrial and academic research groups. Most notably, selective inhibitors of histone lysine methyltransferase EZH2, DOT1L and histone demethylase LSD1 have entered into human clinical trials for the treatment of non-Hodgkin lymphoma, acute leukemia, acute myeloid leukemia and relapsed/refractory small cell lung carcinoma, respectively (27-32). Nevertheless, selective inhibitors for many histone methyltranferases still remain unavailable.

As a member of the NSD histone lysine methyltransferase family, NSD3 dimethylates and trimethylates histone H3 at lysine 36, a conserved epigenetic marker regulating gene transcription, DNA replication and repair, DNA methylation, and alternative splicing that are dysregulated in cancer. NSD3, also known as Wolf-Hirschhorn syndrome candidate 1-like 1 (WHSC1L1), is located within chromosome 8p11-12, a recurrent target region of genetic alterations such as DNA amplification, chromosomal breaks and loss. Abnormal expression of NSD3 in a number of cancer types has been demonstrated (18-20).
Nonetheless, the biological roles of NSD3 in human carcinogenesis are not clear and remain to be clarified.

In the present study, we elucidated the biological importance of histone methyltransferase NSD3 in human osteosarcoma. Our studies revealed that depletion of NSD3 in osteosarcoma cell lines inhibited cell proliferation and survival, and induced cell apoptosis. Furthermore, marked and consistent increases in the population of G2/M phase after RNAi knockdown of NSD3 were observed in all three analyzed osteosarcoma cell lines, probably due to $\mathrm{G} 2 / \mathrm{M}$ cell cycle arrest, indicating the involvement of NSD3 in the cell cycle process. However, the detailed mechanisms of the role of NSD3 in cell proliferation, cell cycle, and apoptosis remain poorly understood.

A key to understand the role of NSD3 in cancer may be through its regulated genes. Hence, a series of NSD3associated genes were identified by RNA-seq, including 244 upregulated and 305 downregulated genes after NSD3 deletion, implying that NSD3 functions as either a transcriptional activator or a repressor. Analysis of Gene ontology (GO) term enrichment revealed that NSD3 negatively regulated a variety of genes involved in the process of negative regulation of signal transduction as well as negative regulation of signaling, negative regulation of cell communication, and negative regulation of response to stimulus, implying the oncogenic functions of NSD3 in human osteosarcoma. GO categories including regulation of cell cycle (GO:0051726), cell cycle arrest (GO:0007050), regulation of cell cycle checkpoint (GO:1901976), cell cycle G2/M phase transition (GO:0044839) and G2/M transition of mitotic cell cycle 
(GO:0000086) were enriched in the differentially expressed genes with depletion of NSD3, implying the crucial functions of NSD3 in the G2/M transition of the cell cycle process. Additionally, a set of selected candidate genes likely to be involved in the development and progression of osteosarcoma was validated by quantitative real-time PCR. Taking these findings into account, NSD3 may function as an oncogenic driver in osteosarcoma. Thus, NSD3 may be a novel target for the treatment of NSD3-associated cancers, and it appears feasible to develop inhibitors of NSD3 for cancer therapy.

\section{References}

1. Ottaviani G and Jaffe N: The epidemiology of osteosarcoma. Cancer Treat Res 152: 3-13, 2009

2. Bielack SS, Kempf-Bielack B, Delling G, Exner GU, Flege S, Helmke K, Kotz R, Salzer-Kuntschik M, Werner M, Winkelmann W, et al: Prognostic factors in high-grade osteosarcoma of the extremities or trunk: An analysis of 1,702 patients treated on neoadjuvant cooperative osteosarcoma study group protocols. J Clin Oncol 20: 776-790, 2002.

3. Botter SM, Neri D and Fuchs B: Recent advances in osteosarcoma. Curr Opin Pharmacol 16: 15-23, 2014.

4. Gatta G, Botta L, Rossi S, Aareleid T, Bielska-Lasota M, Clavel J, Dimitrova N, Jakab Z, Kaatsch P, Lacour B, et al; EUROCARE Working Group: Childhood cancer survival in Europe 1999-2007: Results of EUROCARE-5 - a population-based study. Lancet Oncol 15: 35-47, 2014.

5. Kempf-Bielack B, Bielack SS, Jürgens H, Branscheid D, Berdel WE, Exner GU, Göbel U, Helmke K, Jundt G, Kabisch H, et al: Osteosarcoma relapse after combined modality therapy: An analysis of unselected patients in the Cooperative Osteosarcoma Study Group (COSS). J Clin Oncol 23: 559-568, 2005.

6. Chen X, Bahrami A, Pappo A, Easton J, Dalton J, Hedlund E, Ellison D, Shurtleff S, Wu G, Wei L, et al; St. Jude Children's Research Hospital-Washington University Pediatric Cancer Genome Project: Recurrent somatic structural variations contribute to tumorigenesis in pediatric osteosarcoma. Cell Reports 7: 104-112, 2014.

7. Kuijjer ML, Hogendoorn PC and Cleton-Jansen AM: Genomewide analyses on high-grade osteosarcoma: Making sense of a genomically most unstable tumor. Int J Cancer 133: 2512-2521, 2013.

8. Morishita M, Mevius D and di Luccio E: In vitro histone lysine methylation by NSD1, NSD2/MMSET/WHSC1 and NSD3/WHSC1L. BMC Struct Biol 14: 25, 2014

9. He C, Li F, Zhang J, Wu J and Shi Y: The methyltransferase NSD3 has chromatin-binding motifs, PHD5-C5HCH, that are distinct from other NSD (nuclear receptor SET domain) family members in their histone H3 recognition. J Biol Chem 288 4692-4703, 2013.

10. Li Y, Trojer P, Xu CF, Cheung P, Kuo A, Drury WJ III, Qiao Q, Neubert TA, Xu RM, Gozani O, et al: The target of the NSD family of histone lysine methyltransferases depends on the nature of the substrate. J Biol Chem 284: 34283-34295, 2009.

11. Rahman S, Sowa ME, Ottinger M, Smith JA, Shi Y, Harper JW and Howley PM: The Brd4 extraterminal domain confers transcription activation independent of pTEFb by recruiting multiple proteins, including NSD3. Mol Cell Biol 31: 2641-2652, 2011.

12. Simon R, Richter J, Wagner U, Fijan A, Bruderer J, Schmid U, Ackermann D, Maurer R, Alund G, Knönagel H, et al: Highthroughput tissue microarray analysis of $3 \mathrm{p} 25$ (RAF1) and 8p12 (FGFR1) copy number alterations in urinary bladder cancer. Cancer Res 61: 4514-4519, 2001.

13. Balsara BR and Testa JR: Chromosomal imbalances in human lung cancer. Oncogene 21: 6877-6883, 2002.

14. Ray ME, Yang ZQ, Albertson D, Kleer CG, Washburn JG Macoska JA and Ethier SP: Genomic and expression analysis of the 8p11-12 amplicon in human breast cancer cell lines. Cancer Res 64: 40-47, 2004.

15. Tonon G, Wong KK, Maulik G, Brennan C, Feng B, Zhang Y, Khatry DB, Protopopov A, You MJ, Aguirre AJ, et al: Highresolution genomic profiles of human lung cancer. Proc Natl Acad Sci USA 102: 9625-9630, 2005.
16. Yang ZQ, Liu G, Bollig-Fischer A, Giroux CN and Ethier SP Transforming properties of $8 \mathrm{p} 11-12$ amplified genes in human breast cancer. Cancer Res 70: 8487-8497, 2010.

17. Mahmood SF, Gruel N, Nicolle R, Chapeaublanc E, Delattre O, Radvanyi $\mathrm{F}$ and Bernard-Pierrot I: PPAPDC1B and WHSC1L1 are common drivers of the 8p11-12 amplicon, not only in breast tumors but also in pancreatic adenocarcinomas and lung tumors. Am J Pathol 183: 1634-1644, 2013.

18. Kang D, Cho HS, Toyokawa G, Kogure M, Yamane Y, Iwai Y, Hayami S, Tsunoda T, Field HI, Matsuda K, et al: The histone methyltransferase Wolf-Hirschhorn syndrome candidate 1-like 1 (WHSC1L1) is involved in human carcinogenesis. Genes Chromosomes Cancer 52: 126-139, 2013.

19. Angrand PO, Apiou F, Stewart AF, Dutrillaux B, Losson R and Chambon P: NSD3, a new SET domain-containing gene, maps to $8 \mathrm{p} 12$ and is amplified in human breast cancer cell lines. Genomics 74: 79-88, 2001.

20. Saloura V, Vougiouklakis T, Zewde M, Kiyotani K, Park JH, Gao G, Karrison T, Lingen M, Nakamura Y and Hamamoto R: WHSC1L1 drives cell cycle progression through transcriptional regulation of CDC6 and CDK2 in squamous cell carcinoma of the head and neck. Oncotarget 7: 42527-42538, 2016.

21. French CA, Rahman S, Walsh EM, Kühnle S, Grayson AR, Lemieux ME, Grunfeld N, Rubin BP, Antonescu CR, Zhang S, et al: NSD3-NUT fusion oncoprotein in NUT midline carcinoma: Implications for a novel oncogenic mechanism. Cancer Discov 4 : 928-941, 2014.

22. Shen C, Ipsaro JJ, Shi J, Milazzo JP, Wang E, Roe JS, Suzuki Y, Pappin DJ, Joshua-Tor L and Vakoc CR: NSD3-short is an adaptor protein that couples BRD4 to the CHD8 chromatin remodeler. Mol Cell 60: 847-859, 2015.

23. Rosati R, La Starza R, Veronese A, Aventin A, Schwienbacher C, Vallespi T, Negrini M, Martelli MF and Mecucci C: NUP98 is fused to the NSD3 gene in acute myeloid leukemia associated with $\mathrm{t}(8 ; 11)(\mathrm{p} 11.2 ; \mathrm{p} 15)$. Blood 99: 3857-3860, 2002.

24. Taketani T, Taki T, Nakamura H, Taniwaki M, Masuda J and Hayashi Y: NUP98-NSD3 fusion gene in radiation-associated myelodysplastic syndrome with $\mathrm{t}(8 ; 11)(\mathrm{p} 11 ; \mathrm{p} 15)$ and expression pattern of NSD family genes. Cancer Genet Cytogenet 190: 108-112, 2009

25. Jacques-Fricke BT and Gammill LS: Neural crest specification and migration independently require NSD3-related lysine methyltransferase activity. Mol Biol Cell 25: 4174-4186, 2014.

26. Trapnell C, Roberts A, Goff L, Pertea G, Kim D, Kelley DR, Pimentel H, Salzberg SL, Rinn JL and Pachter L: Differential gene and transcript expression analysis of RNA-seq experiments with TopHat and Cufflinks. Nat Protoc 7: 562-578, 2012

27. Knutson SK, Warholic NM, Wigle TJ, Klaus CR, Allain CJ, Raimondi A, Porter Scott M, Chesworth R, Moyer MP, Copeland RA, et al: Durable tumor regression in genetically altered malignant rhabdoid tumors by inhibition of methyltransferase EZH2. Proc Natl Acad Sci USA 110: 7922-7927, 2013.

28. Knutson SK, Kawano S, Minoshima Y, Warholic NM, Huang KC Xiao Y, Kadowaki T, Uesugi M, Kuznetsov G, Kumar N, et al: Selective inhibition of EZH2 by EPZ-6438 leads to potent antitumor activity in EZH2-mutant non-Hodgkin lymphoma. Mol Cancer Ther 13: 842-854, 2014.

29. Basavapathruni A, Olhava EJ, Daigle SR, Therkelsen CA, Jin L, Boriack-Sjodin PA, Allain CJ, Klaus CR, Raimondi A, Scott MP, et al: Nonclinical pharmacokinetics and metabolism of EPZ-5676, a novel DOT1L histone methyltransferase inhibitor. Biopharm Drug Dispos 35: 237-252, 2014.

30. Klaus CR, Iwanowicz D, Johnston D, Campbell CA, Smith JJ, Moyer MP, Copeland RA, Olhava EJ, Scott MP, Pollock RM, et al: DOT1L inhibitor EPZ-5676 displays synergistic antiproliferative activity in combination with standard of care drugs and hypomethylating agents in $M L L$-rearranged leukemia cells. J Pharmacol Exp Ther 350: 646-656, 2014.

31. Waters NJ, Smith SA, Olhava EJ, Duncan KW, Burton RD, O'Neill J, Rodrigue ME, Pollock RM, Moyer MP and Chesworth R: Metabolism and disposition of the DOT1L inhibitor, pinometostat (EPZ-5676), in rat, dog and human. Cancer Chemother Pharmacol 77: 43-62, 2016.

32. Mohammad HP, Smitheman KN, Kamat CD, Soong D, Federowicz KE, Van Aller GS, Schneck JL, Carson JD, Liu Y, Butticello M, et al: A DNA hypomethylation signature predicts antitumor activity of LSD1 inhibitors in SCLC. Cancer Cell 28: $57-69,2015$. 